\title{
Die finanzierung des wohlfahrtsstaats - eine kleine ortsbestimmung an hand der theoriegeschichte
}

Citation for published version (APA):

Backhaus, J. G. (1989). Die finanzierung des wohlfahrtsstaats - eine kleine ortsbestimmung an hand der theoriegeschichte. Maastricht University. https://doi.org/10.26481/spe.19891110jb

Document status and date:

Published: 10/11/1989

DOI:

10.26481/spe.19891110jb

Document Version:

Publisher's PDF, also known as Version of record

\section{Please check the document version of this publication:}

- A submitted manuscript is the version of the article upon submission and before peer-review. There can be important differences between the submitted version and the official published version of record.

People interested in the research are advised to contact the author for the final version of the publication, or visit the DOI to the publisher's website.

- The final author version and the galley proof are versions of the publication after peer review.

- The final published version features the final layout of the paper including the volume, issue and page numbers.

Link to publication

\footnotetext{
General rights rights.

- You may freely distribute the URL identifying the publication in the public portal. please follow below link for the End User Agreement:

www.umlib.nl/taverne-license

Take down policy

If you believe that this document breaches copyright please contact us at:

repository@maastrichtuniversity.nl

providing details and we will investigate your claim.
}

Copyright and moral rights for the publications made accessible in the public portal are retained by the authors and/or other copyright owners and it is a condition of accessing publications that users recognise and abide by the legal requirements associated with these

- Users may download and print one copy of any publication from the public portal for the purpose of private study or research.

- You may not further distribute the material or use it for any profit-making activity or commercial gain

If the publication is distributed under the terms of Article $25 \mathrm{fa}$ of the Dutch Copyright Act, indicated by the "Taverne" license above, 


\section{DIE FINANZIERUNG DES WOHLFAHRTSSTAATS \\ Eine kleine Ortsbestimung an Hand der Theoriegeschichte}

von

Professor Dr. Jürgen G. Backhaus

Maastrificht, den 10. November 1989 
Dlese Ausgabe ist nicht für den Buchhandel bestimit. Bezug nur durch Uberwefsung won DM 5; - auf das Konto der Gemeinnäzigen Geselischaft fir Stats - und Sozial-Wissenschaftliche Forschungen mbH in Talheim/Neckar, Postgiroamt Stuttgart, Kontonumier 4802-706. 
Die Finanzlerung des Wohlahrtsstats

Enme kleine Ortsbesthmung an Hand der Theor inegeschlchte 1

\section{Zur Einführung}

I. Die naturrechtlichen Vorstellungen von Wohlfahrtsstat

II. Dite Rameralisten

II. Die Physiokraten

IV. Schuldenpolitik, offentliches Bankwesen und die öffentliche Hand a.15 Unternehmer

V. Schlussfolgerung

\section{Zur Einführung}

Wer sich vor die Aufgabe gestellt sieht, sein Lehramt als erster" anzutreten, dazu noch an einer neuerrichteten fakultät, die thr Bestehen einem besonderen Auftrag verdankt, hat allen Anlass, sich die Frage vorzulegen, wie der Auftrag am besten in Forschung und Lehre zu werwirklichen sei. Dies gllt ganz besonders, wenn, whe fin melmem Fall, der Lehrauftrag umfassend beschrieben ist, das Fach uber eine mehrhundertjährige Tradition verfügt, sodass unterschiedliche Wege efm geschlagem werden können, und dle äusseren Bedingungen es nahelegem, Finanzwissenschaft Im Hinblick auf Anwendungen in ginem entwickelten Wohlfahrtstat zu betreiben, der nicht allein den grössten Telli, sondern weit mehr als die Hälfte des gesamten sozlalprodukts direkt verausgabt und auch die Verwendung des übrigen Volksefnkomens in vielfältiger Weise beeinflusst. 2 Als weiterer Ausgangspunkt dieser Dberlegungen komt noch hinzu, dass die Gründungsvatter dieser Fakultät besonderen wert darauf legten, dile verschledenen wirtschaftwissen-

1 Offentliche Vorlesung, die der Verfasser anlasslich des Antritts des Antes elmes ordentllichen Professors der Flnanzwissenschaften an der Refchsunfversitut 1 imburg am 10. November 1989 im Masstricht gehalten hat. Der Vortragstil wurde belbehalten.

2 Die Grafik (vergleliche Selte 2 des nlederlandlichen Textes) zeigt. dass dle Niederlande und Schweden an dar Spltze der entwickelten Staten mit hohen Stadts(ausgaben)quoten stehen, watrend die Schwelz (verglelche die tabelle auf selte 3 des niederiand schen Textes) und japan mit halb so grosisen statisausgaben Betsplele fur elnen anderen Wirtschaftsstll abgeben. Auf die Schwelz wird hler vor allem deshalb verwilesen, well sle mit ihrer geschichtilichen Entwickiung in der westlichen Traditlon steht und lhren turgern zweifellos eln hohes Wohlfahrtsniveau garantilert. 
schaftlichen Diszlplinen, vor allem die Volkswirtschaftslehre und die Betrlebsillitschaftslehre. in Forschung und Lehre so weft wie möglich zusammenzufühen und Wirtschaftswissenschaftler fur Forschung. Lehre und Praxis auszubliden, dfe an Hand von Problemen lemend praktische Fragen in der hirklichkelt thres späteren Berufs auch sinnvoll zu lösen in der Lage sind.

Diese Randbedingungen bestimen den Aufbau des Vortrags. Als Ausgangspunkt wählle ich die erste für unser fach wirksame wissenschaftliche Bestimmung einer Lehre des Wohlfahrtsstates, zeige, wie sie zu elner praktlschen Fynamzwissenschaft ausgebaut werden kannte, und verfolge den weiteren Entwlcklungsgang der Finanzwissenschaften an Hand zwefer wichtiger statilicher Polltikinstrumente, die nach und nach hinzukamen: der Akzlse als besonderer Stewerform und des statilichen Bank- und Unternehmenswesens. Damlt sind zwel Eckpfeiler der Finanzferung des modernen Wohlfahrtsstaates bereits benannt, die díe Praxis entwickelt hat. Aber auch die Theorle hat auf das Fach einen grossen Einfluss ausgeibt. An Hand eines Beispleles, der Physlokraten, zetge $1 \mathrm{ch}$, welche Möglichkeiten und Probleme stch ergeben, wenn man ein theoretisch geschlossenes Modell auf die Praxis der Staatsfinanzen anwenden will. Schlfesslich bespreche ich noch die Frage, welche Stränge in der vielfältigen Tradition der Finanzwissenschaften für das Fach besonders vielversprechend sind wenn sich der Finanzwissenschaftler in der Praxis des modernen Wohlfahrtsstaates mit seinen vielfältigen Aufgaben und Problemen bewähren muss.

Dle naturrechtlichen Vorstellungen vom Wohlfährtsstaat

Die naturrechtliche Begründung des modernen Wohlfahrtsstaates und glefchzeitig die Begründung der Statslehre verdanken wir Christian (Freitherr von) Wolff (1679-1754). Selt 1707 Professor der Mathematik, Phllosophie und Naturlehre in Halle, gelang es Wolff, im Wege seiner streng mathematisch demonstrativen Methode auf der Leiboizschen Philosophle aufbauend diese um den sittilich praktischen Teil systematisch zu erweltein und so die Grundlagen für die moderne Finanzwlssenschaft zu legen. Dazu bedurte es zunächst des Nachweises, dass eine staatsWlssenschaft mit praktischen Anwendungen überhaupt möglich sein könne. Wir können diesen Beweis hter überspringen und uns gleich seiner praktischen Aufgabenstellung ain den Staatswissenschaftler zuwenden. Ste 
beschrefbt noch heute zutreffend die praktische rätigkeit des Finanzwissenschaftlers, und findet sich noch wortlich zum Beisplel in dem Auffgabenstell ungen der Rechnungshöfe.

5 4. Nachdem es nun bekannt 1st, dass die Enwegung der Staatsgeschäffte nichts urmögliches sey $(\ldots .$.$) : so gebïhret es sich nun$ dieselbige in etwas genauer zu betrachten. So offt man wegen der Staatsgeschäfte zu Rath gehet, so kormen zweyerley Fragen vor. Die eine Art derselbigen betrifft das Recht, ob man nehmllich eine Befugniss zu etwas habe, und es ohne dem Rechte eines anderen zu nahe zu treten thun könne oder nicht; die andere betrffft den Nuzen ob es nehmlich einen vorthefl bringe, dergleichen zu thun oder nicht. 3

Neben der Rechtnässigkeft einer Massnahme ist vor allem die Zweckmässigkett, die ökonomische Wützl lichkeit oder Effizienz zu prüfen.

Interessanterweise begegnen wir derselben Zweiteilung ebenfalls in der modernsten Finanzwissenschaft, wo neben Fragen der allokativen Efflzienz zunehmend Fragen der Verteilungsgerechtigkeit (equity) eline Ralle splelen, die abgelöst von der konkretem Rechtsordnung erörtert werden. Daraus ergibt slch die Gefahr elnes Konflikts zwischen der tatsächlich vorgefundenen Rechtsordnung und Jenen der Wohlfahrtstheorfe entlehnten Gerechtigkeitswarstellungen, wenin die Ergebnisse der finanzwissenschaftlichen Arbeit praktisch ungesetzt werden sollen. Wolff geht auf dieses Problem direkt ein, Indem er auf die Zusammengehörigke1t der juristischen und ökonomischen Analyse für die Zwecke der Wirtschaftspolitik pocht:

Es müssen demnach diejenigen, welchen die Sorge pür die Staats geschäffte aufgetragen ist, zwey Tugenden vor anderen besizen; nehmlich die Gerechtigkeit und Klugheit. Jene haben sio nöthig damit sie nichts so thun verlangen noch dazu rathen dass man etwas thue, wenn es nicht gerecht ist, und damit sie war der Ungerechtigkelt elnem Abscheu haben: Dlese aber wird dazu erfordert, damit ste kelnen Schaden anrlchten, indem ste Nuzen schaffen wollen

3 Christlan Fretherr won Wolff, "Won einer Erwegung der Staatsgeschäfte". In: Gesammlete kleine philosophische Schrifften. Halle: Renger, $1740,5,372-373$. 
(ebenda).

Der grosse Erfolg. dessen sich die okonomfsche Rechtsanalyse in den letzten Jahren erfreut, beruht auf der Widerwerefnigung dieser beiden Aspekte in der polftis sch-ökonomischen Analyse.

Auf dle Notwendigkelt, den akademischen bnterricht an den praktischen Problemen zu orfentferen, wefst Wolff uns ebenso hin wie darauf, dass dife vorhandenen Lethrbicher unter diesem Aspekt kritisch zu gebrauchen sind. 4

Mann lehret ja die Staatskunst in keiner andern Absicht, als damit mann lerne; was den Staat zuträglich seye, und demnach dazu müzlich seye, den staatsgeschäfften klüglich vorzustehen. onnerachtet nun diejenlge Staatskunst, welche gemeinlgifch in den Schulen gellehret wird, solchen gair wenlg nüzlich ist, welche mit elner Staatsklugheit versehen seyn müssen: so kan man jedoch nicht In Abrede seyn; dass in den Büchern verschledener Schriftsteller, welchen man thr Lob nicht nehmen kan, manches vorkomet, was dabel nizlich ist, wenn man einen Lehrbegriff aufsezen wi11.5

Schllesslich finden wir noch den wesentlichen Hinweis, dass man neben der Theorile auch deren Anwendung lernen müsse ( $\$ 10)$, und dass eine bestimmte theoretische Erwägung kaum sinnvoll angewandt werden könne, wenn man nlcht die konkreten umstände dfeser Anwendung ebenfalls ausführlich mitbericksichtigt (ebenda).

Soweit Wolffs Methode. Und was sagt er zu den Inhalten? Wir finden die Grundlegung seiner Staatsw1ssenschaft eingebettet in sein Staatsrecht: in der zweiten Abtellung des dritten Tells seines völkerrechts in sechs Kapitein. Wesentilich ist die für thn charakterlstische Bestimung des Statszwecks als Wohlfahrtsstaat.

4 Betde Geslchtspunkte versuchen wir in Mastirfchter Lehrsystem zu verwlrklichen, indem der Unterrlcht problemorlentlert aufgebaut wird und die studenten asdrucklich dazu aufgefordert werden, slch an Hand der Pür das Problem geelgneten wisenschaftichen Literatur, anstelle elnes vorgeschriebenen Lehrbuches, zu orientieren. 
\$ 972. Wir erkennen sehr leicht, dass eintzele Häuser sitch selbst dasjenige nicht hinrefichend verschaffen können, was zur Nothdurft, Bequemlfchkeit und dem Vergnügen, ja zur Glückseligkelt erfordert wird, noch auch thre Rechte ruhig gentessen, und was ste von andern zu fordern haben, sicher erhalten, noch auch steh und das ihrige wider anderer Gewalt thätlgkelt schützen können. Es ist also nöt1g, dasjenige durch gemelinschaftliche Kräfte zu erhalten, was eintzele Häuser vor sfch nicht erhalten können. Und zu dem Ende mïssen Gesellschaften errichtet werden $(5836) .6$

Man beachte, dass Wolff nicht von Individuen, sondern von Haushalten a1s den Kleinsten wirtschaftilchen Entscheidungseinheiten ausgeht. Was die Haushalte selbst nicht zu threr eigenen wohl fahrt für sich erhalten kömnen, wird Aufgabe des Staates. Trotzdem, und für die modernen Diskussionen rundum den Wohlfahrtsstaat nicht unwichtig, sind der Statsgewalt wesentliche Schranken auferlegt.

\$980. Da dile Herrschaft in einem State aus seiner Absteht ermessen werden muss ( 976,972 .); so erstreckt sle stch nlicht welter als auf die Handlungen der Bürger, welche zur Beförderung der gemeinen Wohlfahrt gehören; folgltich da nur bloss in Absicht dieser Handlungen die natürliche Freyhelt der einzelen Glleder eingeschränckt wird $(\$ 975,77$.$) ; so bletbt sle in Ansehung der$ übrigen Handlungen ungekränckt. 7

Die Staatsgewalt refcht nicht weiter als jenes klar umrissene staatsziel, das seine Begrenzung in der wirtschaftlichen Funktionsfähigkel t der Haushalte findet. Die Hauptaufgabe des Wohlfahrtsstaates besteht dann darin, die notwendigen Vorkehrungen zu treffen, um dle Haushalte in die Lage zu versetzen, thren Lebensunterhalt selbst zu bestrelten.

1021. Auf dass was zum Leben erfordert wird hinlängllch da sey, sa 1 st nöthig, dass die Wercke des Flefsses und der Kunst so sehr wervielfältiget werden, als es möglich ist, damit nicht dlejenigen

6 Chrlstyan Fretherr won Walff, Grundsätze des Natur- und völckerrechts. Halle: Renger, $1754,11 \%, 2.1$.

7 Wolff, 1754, III, 2.1. 
milissig gehen durfen, welche Krafte zum Arbetten haben, und es denen nch an Arbett fehle, die Arbelten wollen, dass dergestalt eln Jeder durch selnen Flelss und Arbelt so viel vor slch bringe, als erforderlich 1 st; wo nicht nützlliche und zum Vergnugen gehörigie, doch nothwendige Dinge anzuschaffen (972.): diesen zu folge muss der Werth der Dinge und der Arbelt bestimet, und dafur bescens gesorget werden, dass nicht die Unterthanen in Armuth und Mangel, oder gar an Bettelstab gerathen. Und eben desswegen muss man nicht gestatten, dass Theurung gemachet werde, ja so wiel als es stch thum lassen will, muss mar den Unterthanein Erletchterung schaffen, dass de Theurung then nicht zur Last falle. Wefl auch der Holtzgebrauch ungemein gross und ganitz unentbehrllich ist; so muss man sorgan, dass es nicht an hinlanglichen Holtzvorrath fehle, und daferne es rar werden sollte, so muss man die Unterthanen zum rathligen Gebrauch desselberi anhalten.

Das Z1tat aus 1021 zeigt, dass Wolff berelts drel moderne Zlele der Finanzpolltik betont: die Vollbeschäftigung, "Dass es denen nicht an Arbeft fehle, die Arbeften wollen", die Prefsstabl1ttat "muss man nicht gestatten. dass Theuerung gemachet werde" und die Umweltpolltik, nachdem Raubbau die naturliche Resource Holz, das wesentliche Brennund Baumaterial, cramatisch verknappt hatte.

Die sozlalpolltischen Aufgaben orlentieren sich wiederum an den Prinzip der Funktionsfähigkelt der Haushalte. Wo diese inicht Velsten können; muss der Staat elngrelfen; dann aber über das ganze Spektrum der damals bekannten Wohlfahrtseinrichtungen, von der Armen- und Sozialpflege, den Walsenhäusern, den Beschäftigungs- und Schuluingsprogramen für Arbeltsiose bis hin zur kostenlasen Ausbildung der Kinder armer Eltern.

$\$ 1022$. Das Leben gehörlg hinzubringen, wird auch erfordert, dass män vor dle bürftige und Bettler besarge, was zur Nothdurft des Lebens nothlg 1st, und, damit die Unterthanen nicht gar zu sehr myt Allmosengeben beschwehret werden, ist in sorgfiatlge Betrachtung zu ziehen, was das Naturgesetz von dem Allmosen feste setzt (3 488. seq $_{*}$ ). Daher sind Zuchthauser aufzubauen, wortin diejenigen zur Arbeit angehalten werden müssen, welche, ob ste gleich Arbelten koinnten, doch lifeber betteln wollen; Ingleichen Armen- 
häuser, worinn man die dürtigen ernăhret, dle stch durch Arbelten das nicht zu enwerben in Stande sind, was ste zur Lebensmothouft gebrauchen, und keine Anverwandten oder Freunde haben, wiche stoh Inrer Bedürfilsse annehmen könnten: noch ferner kranckenhaser, worinm krancke Arme thells ernahret, thells gehellet serden: So auch Waysenhauser, worinn man ame Waysen erzlehet: andich Armenschulen, in welchen man die Kinder armer Eltern umsonst in demjenigen unterrichtet, was thnen zu wissen nöthig und nitzilich 15t. 8

Ein derartig weitgespanntes sozlalpalitisches Aufgabenprogranm fuhrt zugleich zur Frage nach dessen Finamzierung. Wolff gibt efinen ganzen Katalog der traditionellen Staatseinkunfte, und warnt soglelch, dass man mit der Erhebung von Zöllen und Steuern und der Auferlegung von Sonderabgaber "behutsam verfahren müse", da dle Steuerm und Sonderabgaben den Wert der besteuerten Sache senken, die zölle aber die Preise erhöhen, sonit beide das Elnkomen der Haushalte senken, 15 1057). Die Akzisen erwähnt er noch nicht.

Wir könnein zusammenfassend den Schluss zlehen, dass Wolff die Stats wissenschaft berelts als eine praxisbezogene Wissemschaft systematisch begrindet und sich mit dieser Wissenschat direkt an Identiflzterbare, handelnde Personen wendet, denen er mit selnem wissenschaftlich begrundeten praktischen Rate zur Selte stehen will.

\section{Die Kameralisten}

Mit den Kameralisten begegnen uns die ersten systematisch arbeitenden Fimanzwissenschaftler. Es wird oft vorgeschlagen, die Kameralwissenschaften in sechs Disziplinen. zu unterschelden, nämllich erstens die Privatökonomie lelnschllessllch der Landwirtschatt, der forstillschaft, der Technologle und der Handelswissenschatt), zwaltens der Pollzelwissenschaft, die man heute Verwaltungswsisenschaft nennen könnte, drittens der Kamera 1 wsenschaft im engeren Simne, der houtlgen Finanzwlssenschaft, viertens der Statyst1k, funftens des Rechnungswesens und sechstens der Kameralpraxis. Her ist wor allam die Kaneralwissenschaft im engeren sinne, also die finanzwissenschaft von Bem 
dewtung d1 1727 nit der Errichtung der ersten wirtschaftsussenschaflichen professur (In Halle) in alsademischen Unterricht vertreten 1st. 1796 gbt es in deutschen sprachraum berelts 23 solcher Lehrstuhle, In oxford wird der erste Lehrstuh fur polltische okonome in Jahre 1826 mit Nasau sentor besetzt.

Q1 Eedeutung der Kamerallstem ersehen wir nicht allein dus diesen Faktum, sondern auch aus mrem Namen, der andeutet, dass ste den Zugang zur Ratskamer der Reglerung besassen. 9 Damit 1 st der Adressat threr Lelusysteme wieder ummltelbar bezelchnet: die auf efgene Verantwortung handelnde Reglerung. Es lst aber unrichtig. die Kamerallsten als dif unkritschen Dlener absoluter Fursten darzustellen, die unablass1g deren Schatzkammern zu fullen slch anschickten; selbst aber ke ine theoretisch-ökonomischem EInsichten besassen. 10 Dle Regierungen Jener Zelt waren oft, nitht Immer, - es gab elne grössere Anzahl von Republiken - absolute Fürstentümer; aber dile Lehre der Kameralisten war dle Wolffsche Lehre des Wohlfahrtstates, fim stnne Jener Glückseligkelt der Staaten, von der oben die Rede war.

Kennzelchnend für die Flnanzwlssenschaft der Kameralisten ist. dass sie in etn sorgrältig abgestimites system der Wirtschaftspolitik eingebettet $1 \mathrm{st}$, dle die Zielle des Hohlfahrtstates nit allen dem State zu Gebote stehenden Mitteln eirrelchen will. Wan begnügt sich bel der Beurtellung einer wirtschaftspolitischen Massnahme nicht darit, die beste wirtschaftliche Has shahme zu bestimmen; oft haben auch andere, $z_{\text {. }} B$. Verwaltungmassnahmen einen wesentilichen Einfluss auf das wirtschaftliche Geschehen. Nicht die relativ bessere, sondern die absolut beste Massnahme aus dem gesamten denikbaren Katalog staatspolltischer Massnahmen ist auszuwählen:

9 Johann Heinrich Gottlob von Just1. Staatswithschart oder Systemat ische Abhandlung aller ökonomischen und Cameralulissenschaften. Lelpztg: Breftkopf, 1758, 1, 3 3: Den Namen der Cameralwissenschaften aber legett man thner deshalb bey, wefl dife hohen Collegla, welche der Landesherr niedergesetzet hat, um dle Geschaffe der Erhaltung, der Vermehrung und des Gebrauchs des Vermögens der Reputilk zu besorgen, gemelniglich Camern oder Cammercollegla geneninet werden.

10 So 2.B. J.J. Klant. Het ontstaan van de staathulshoudkunde, Mulden/Antwerpen: Stenfert Kroese, 1988, Sefte 90 . 
Man kann öfters den Maassregeln eines Regenten und selner Munlster nicht absprechen, dass sie nützlich sind und zu der Wohlfahrt des Staates gereichen. Allein so bald man erwäget wie viel nutzlichere und heflsamere Anstalten, entweder gar wicht unternomen, oder doch schlärig und nachlässıg besorget werden; so fällt aller Verdienst hinweg, den man ihnen vielleicht auf den ersten Anblick bey jenen nützlichen Maassregeln zugestanden hat. 11

Dieser Satz ist bezelchnend, zeigt er doch, dass Justi das gesamte Produktionspotential einer Volkswirtschaft in den Dienst des Wohlfahrtsstaates stellen will, und dabei den effizientesten Einsatz nicht nur der privaten Haushalte und Unternehmungen, der Landwirtschaft und der Gewerbe im Auge hat, sondern auch der staatl tehen Organe.

Das Produktionspotential der Volkswirtschaft (er nennt es "Wermögen") bestimnt er ausserordentilich modern. Zum Vemögen des States gehören alle beweglichen und unbeweglichen Güter, welche im Lande vorhanden sind, mögen sie den Untertanen gehören oder dem staate unmittelbar zustehen. Justi rechnet dazu "auch alle Fähigkeiten und Geschicklichkeiten derer in der Republik befindlichen Personen", also das Humankapital. Die "höchste Gewalt", das heisst die optimale Wirtschaftspolitik, besteht im Gebrauch dieses gesamten Vermögens und der Kiräfte des Staates.12 Dieses breit definierte volkswirtschaftiliche Produktionskapital steht mit dem Zweck des Wohlfahrtstates in einem unverbrülchlichen Zusammenthang, aus dem stch soglelch der oberste Grundsatz der kameralistischen Finanzpolitik ableitet: die Erhaltung und Vermehrung des Staatsvermögens (im oben definierten weiten Sinne).

21. Es kann aber das Vermögen des Staats nicht gebratucht werden, oder wenigstens wird der Gebrauch woin keiner langen Dauser seyn, wemn man nicht zugleich auf dessen Erhaltung bedacht ist. Wielmehr da dle Beförderung der Giluckseligkeft der grosse Endzweck aller Republiken ist: so muss der Regent das Vermógen und ole Kräfte des Staats auf alle Art zu vermehren suchen.

Dass es stch bef diese Vermehrung des Staatsvermögens eben nifcht darum

11 Justi, $1758(2), 11$, S. 16.

12 Just, $1758(2), 1,19$. 
handelt, die schatzkamer zu fullen. wird kurz darauf mit aller

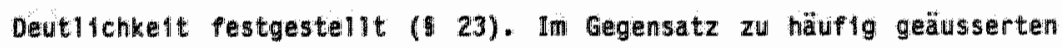
Misverstandnissen des kameralismus (sowle des Merkantllismus) gent es gerade nicht darum, das cold zu horten (es sel denn es musse daraus zu gegebener zeft eln Krlegsschatz zur Bezahlung von söldnerheeren geschlagen werden), sonderm das Augemerk ist darauf gerfchtet, offentllche Infrastruktur-Investitionen und priwate Geschafts- und Hushalstatighett in einem optimalen Masse so aufeinander abzustimen, dass dile produktionsmögllchkeltsgrenze der gesiamten Volkswrtschaft errelcht wird:

Cerlach fasst das wesentiche in enger Anlehnung an die originaltexte so zusammen: 13

D1e Staatse1nkinfte nüssen auf dfe unschädlichste Art crhoben und auf den Gewinst der Untertanen gegründet werden: de Substanz des Vermögens darf nicht angegriffen, daher darf nut elin Tell des Gewingtes den Untertanen entzagen werden. Anderselts darf der staiat auch nlcht zu wenlg beanspruchen: es muss "der gerechte Tell des Gewinnstes, und zwar nlcht mehr, aber auch nicht weniger, zu dem bereltesten Vermögen des Stats wrklich erhoben werden: und 1st es in der That elne unzeltige der Wohlfahrt des Staats gar nicht gemässe Gütigkeft der Reglerung, wenn sie nicht so wiel bereitestes Vermógen gründet, als die Nutzung des gesammten Varmogens der Republik auf elne unnachtelifge Art ertragen könnte. Wenn der Regent wenig Elnikünfte hat, so ist es natürl1ch, dass er viele heflsame Anstalten zu der Wohlfahrt des Staats unterlassen muss. Wenn auch zu wenig Abgaben eingeführt sind, sa nehmen dadurch wiele Unterthanem Anlass, der Neigung zu der Faulheit nechzwhängen. Indem viele es für eime grössere Gückseligkeit halten, nicht zu arbeiten, als Vermogen zu erwerben. Gie Guter der gesammten Republik werden also nicht so genutzet, wie es seyn konnte Uberdiless komt cs bel so wentg Abgaben bloss aut die Nelgung lines Unterthanen an, ob er das Ersparte in dem Kasten

13 otto Gerlach, "Geschichte der Finanzwissenschaft unter besonderer Beruckstchtigung der Lehre vom Verhältnis zwischen Volkswirtschaft. Staat und Flnanzen". In: Dle Entwicklung der deutschen Volkswirtschaftslehre in neunzehnten Jahrhundert (Festschrift Gustav Schmoller), II, Lelpzig: Duncker \& Humblat, 1908, Kap. 38, S. 6.7. Gerlach zitlert aus Justis Staatswirthschaft, $1758(2)$. 
müssig liegen ader in den Gewerben cirkulieren lassen wll. Dahingegen eln weiser Regent und seine Casse gletchsam das grosse Meer ist, in welches ein grosser Tefl des baren Geldes lllesst, und welches sich hinwiederum durch wahl elngerichtete Ausgaben und Reglerungsanstalten über alle Telle des Nahrungsstandes erglesst. und allenthalben elnen blühenden Zustand der Gewerbe und dle Cirkulation des Geldes werbreltet."

Da offensichtilich hier den Steuern auch elne volkswlrtschaftliche Funktion beigemessen wird, Ist wiederum der Hinwels whichtig, dass dfe Erhebung der Steuern auf die schonendste Art $2 u$ erfolgen hat. Dies folgt direkt aus dem Staatszweck der alygemeinen Wohlfahrt (Giuckseligkeit). Der Grundsatz wird ubrigens wieder radikal gehandhabt. Nicht nur soll elne bestimte Steuer so schonend wie möglich erhoben werden; das Schonungsprinzip wird auf alle Steuern komparativ angewendet:

Allein, eine Art der Erhebung ist doch imer den Commerclen, dem Nahrungsstande und der Freyheit der Unterthanen, mithin threr und des Regenten gemeinschaftlichen Wohlfahrt. schädlicher, is die andere. 14

Man beachte, dass Justi den Zusammenhang zwischen Bürgerfreiheit und Besteuerung sofort in die Steuergrundsabtze aufnimmt. Dyes ist nicht allein ein Uberbleibsel aus der wolffschen naturrechtlichen Grundlegung der Staatswissenschaften; bei Justi kommt der bürgerlichen Frelheit noch deshalb besondere Bedeutung $z u$, weil der kameralistische Staat stets in Konkurrenz mit anderen steht und deshalb micht nur für seine Bürger, sondern auch für unzufriedene Bürger anderer staten so attraktiv wie möglich sein muss. Die Polltik-Instrumente, die der Bevölkerungspollttk dienen, sind freflich nicht finanzwissenschaft11 icher Art; deshalb steht bet den Kamerallsten neben der Flnanzwissenschaft stets die verwaltungswissenschaft; belde sind untrennbar miteinander verbunden. Whe das Problem angepackt wird, sehen wit auch

14 Justi, Staatswirthschaft, II, $1758,16$. 
bef Just in desen Policey-w1stenschatt (Venaltungswissenschaft), worth das zwelke Buch des ersten Tells dem Problen "der Vermehrung der Elfwohner gewfinet ist. Die Elnwohner sind de Trager des Humankaptals und sind deshalb fur de Witchaftspolltik won grosser Bedeutung. Das Buch besteht aus vier Abschniten, die konsequent vier Haptaspekte behandeln. Vorassetzung fü Jedwede Bevölkerungspolitik sind Werlasslche Bevolkerungstatstiken. Thnen ist der erste Abschnlt gewfinet. Der zwelte Abschntt enthält die allgemeinen Grundsätze der Bevolkerungspollth, dfe darau hinasisufen, ein liberales, famlllenfreundliches klima zu schaffer. "Nichts lst in er Tat der

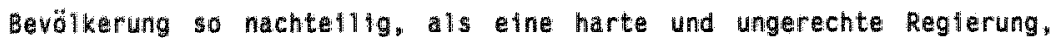
welche dile Unterdruckerin und die Tyrannin thres Valkes ist (Selte 206): und " "die zwelte crundregel der Reglerung muss dennach sein. den Ehestand zu befórdern, und denseiben in Ehren zu halten. Ste muss folglich affmerksam sein, dass keine Grundsatze, Meinungen und Helgumgen Im State Hurzell schlagen, welche den Ehestand in Verachtung brimgen, oder eine Abneigung wor demselben erwecken (Selte 207)." Es folgen sehr praktische Massnahmen, z.B. die Elnrichtung won staatilichen Sparkassen, un "armen Mägden" die Ersparung elner Mitglft zu ermöglichen. Der dritte Abschnitt beschäftigt sich mit der Möglichkeit, das Land zu efinem Elnwanderungsland auszugestalten; und der vierte damit, dem Bevölkerungsschwund vorzubeugen, efrnal durch dle statiliche Gesundheltsfürsorge, un der Morbidität entgegenzuwirken; zum anderen dadureh, dass ketn Amlass zur Auswanderung gegeben wird. Endlifh ist es thm um den Unterhalt des Volkes zu tum, nämllch dass ausrelchend Grundnahrumgsmittel zur Verfüunng stehen, sodass die Bevölkerung weder unter Knapphelt noch $z u$ hohen Preisen für Grundnahrungsmittel zu leiden hat.

El the besondars wichtlge findinztechnische Newerung verdanken wir auch den Kamerallsten: die Akzusen. Der Steuerprakttker Chrtistian Tenzel (allas Teutophlius) beschrelbt deren Vorzuge blldhaft in den ausfühllowen Tltel setnes Buchus

Entdeckte Goldarube in der Accise, Das Ist, Kurzer ledoch

15 Wohan Hefinrich Gottlob won Justi, Dle Grundfeste zu der Macht und Glücksellgkelt der Staaten; oder ausfuhrliche Wortesung der gesamiten Pollcey-Wissenschaft. Ronigsberg und Leipzig: Hartung, I 1760, II 1761. 
Gründlicher Bericht won der Acclse, Dass dieselbe ficht allein die allerretcheste, sondern auch pollteste, billlgste. und nützlichste, ja eine gantz nötige collecte, und also Zwtefacher Ehren werth sey, Darinnen zur Genüge angeführet, warumb die Accise in Deutschland durchgehends, und auff was Arth dleselbe Anfangs in einer Provinz oder Stadt, in deren Nachbarschafft die Accise nicht, sondern die contribution in schwange, elnzuführen, In Sonderheit aber behauptet wird, dass dieselbe das rechte Fundament zu der neuesten Polftique und Comercien-Beförderung lege, Wornebst auch comparative Van der Contribution und Schatzung etwas beygefüget ist, Allen obrigkeiten zu grossen Auffnehmen, und populirung Inres Landes, denen Staats - und Cammer-Bedienten "zur Verminderung Ihrer Geld-Sorge, und denen Unterthanen zur Erleichterung Inrer Last Worgestellet. 16

Die Akzise bot in der Tat eine Reihe von Vortellen. Sie war ergleblg, wie der Ausdruck "Goldgrube" besagt. Ste war eine (im Verglelch) schonende Form der Besteuerung, die bef sinnvoller Handhabung praktysch kelne negativen Angebotseffekte haben musste. Dadurch, dass messbare Konsumausgaben besteuert wurden, entfiehl das bei Einkomensstewern problematische schätzproblem. Der politische vorzug schliessifich bestand darin. dass die Akzisen durch die Stände nicht gemehmigt werden mussten, thre Einführung somft die Entstehung organisierter stat11 cher Organe, die Entstiehung des modernen Staates überhaupt, wesentlich erieichterte.

Wir können abschllessend festhalten, dass die Kameralisten bereits in systematischer fonm die wesentlichen ökonomischen schlussfolgerungen aus dem Wohlfahrtsstaatsgebot gezogen haben, und diese sowohl auf die Steuer als auch auf die Sozlalpolitik im weiteren Simne angewendet haben. Besonders indrucksvoll ist wederum, dass dle Haushalte als kleinste produzterende Einhelten thr besonderes Augenmerk verdtenen; und dass meben einer modern anmutenden 1 lquiditätsorfentfarten Finanzpolitik vor allem auch das Humankapital als Produktfonsfaktor elne

16 Zerbst: Johannes Lüderwaldt, 1685. Verglefche auch Gustav Schmoller, Die Epochen der Freussischen Finanzpolltik. Schmollers Jahrbuch, N.F. 1, 1877 und Frltz Karl Mann, Steuerpolttische Ideale, Jena: Gustay Fischer, 1937, Nachdruck: Stuttgart, 1978 Kap. 4 . 
grosse holle splelt, de durch geelgnete finamz- und sozlalpolltisthe Massnafmen noch stimulifert wid.

\section{Die Phys lokriaten}

Der Beglin der theoretischen Finanzwissenschaft wird oft befi den Physiokraten gesucht ole aus den Kreislaufschema (tableau economique) aboleltete steveriehre (1mpot unfque) bletet dazu den Anias. Die Phys lokraten stelien in der Tat eine kaum zu unterschatzende kraft fü de Ausarbeltung ther theoretischen Volkswirtschaftslehre dar; könmen mit Fug und Recht davon sprechen, dass sie die erste schulle in der Volkswirtschaftslehre blideten, der allerdings nur eine kurze Lebensdauer (von etwa zwel Jahrzehnten) beschieden war. Der elgentllich finanzwissenschaftliche Beitrag. 117mpôt unfque, blieb Konzept 17 und doch gab es vlelfältige Versuche, die Lehren der Phystakraten in finarizolitischer Absicht umzusetzen. Ich möchte an einem Befspiel demonstrferen, zu welchen problemen es dabel komen konnte.

Wir hatten oben festgehalten, dass es stets darum gehen müsse, ein theoratisches Konzept auch efmer prakt1schen Anwendung zuzuführen, und gegebenenfalis im Wege eines Experimentes die Taugltchkett des theoretischen Konzeptes zu erproben. Erstaunllcherweise finden wir bei den Physlokraten eine deutliche Imurisierungssitrategie gegen diesen Test. Der Zusammenhang ist der folgende.

oft wird behauptet, das tableau économique mit seinem kreislaufschema sel elne Analogle des Blutkreislaufes, und darin bestehe die originäre Lelistung des Arztes Quesmay. Nun war Francols Quesnay (1694-1774) zwelfellos Arzt, und auf selner Stellung ais Hofarzt der Madame de pompadour beruhte sein polltischer Eintluss. Das Krefisiofschena als Analogle zur Blutzirkulation wurde freflich von Justl in die wirt-

17 Das Aufsehen erregende Expertment carl Frledrichs von Baden Durlach, der zwischen 1770 und 1771 in den drel Dóffern Dlet1lingen. Thenlingen und Ballingen alle Abgaben durch alne elinzlge Grundsteuer ersatzte, schlug fehl. Das Experiment wurde 1792 entguittig abgebrochen. Vergletche H.P. Liebel, Enlightened Burcaucracy versus enllghtened Despotism in Baden, 1750-1792". In: Transactlons of the American Phllosophlcal Society. N.S. 55, 1965. 5. 40. 
schaft5wissenschaftliche Literatur eingefuhit. 18 Die Analogie, die Quesnay wählte, war nicht der Blutkreislauf elnes menschlichen Organfsmus, sondern vielmehr ist Quesnays Krefslaufblld eine Uhrenanalogie im sinne der Kartesianischen Mechanik seiner Zeit.19 zu dieser Uhrenanalogie gehört auch die Vorstellung. dass die thr mit elnem Schüssel aufzuztehen sel, der Schlüssel aber nur durch Gott den herrn zu bedienen. Wir dürfen nicht vergessen, dass das Kreislaufblid in eine sehr bestimmte Staatsphilosophie eingebettet war.

Dieser Umstand hatte erstaunliche Folgen, als der Markgraf Carl Firledrich von Baden Durlach sich mit der Bitte um Rat an den Marquis de Mirabeau wandte, um ihn auf folgenden Sachverhalt hinzuweisen. Das physiakratische Rezept sei auf seine Ländereien deshalb nicht anzuwenden, well das fränkische Erbrecht zu einer fortdauernden Tellung der Länderelen führe, die diese zerstückele, sodass die Landwirtschaft zurückbleibe. Ob er, der Marquis, aus physlokratischer sicht empiehle, das Erbrecht zu ändern.

Offensichtilich handelt es sich hier um ein einfaches rechtsäkanomisches Problem, das Car1 Friedrich korrekt gestellt hatte und das in der kameralistischen Tradition selbstverständlfch einer Lösung im Dlenste des Wohlfahrtstaates zugeführt worden ware. Die Antwort des. Marquis de Mrabeau allerdings lautete, dass der Markgraf nicht das Recht habe, das won Gott gegebene Erbrecht zu änderm. Das heisst, der Markgraf durfte den Schlüssel zur Uhr der Wirtschaft nticht benutzen.

Schuldenpolytik, öffentliches Bankwesen und die öffentliche Hand als Unternehmer

Will man die beiden einflussrelchsten klassischen Werke, die die

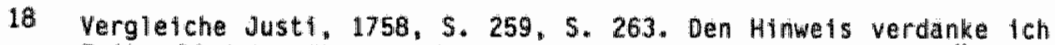
Frltz Blatch, "Der Beltrag der deutschen Physlokraten für dle Entwlcklung der Wirtschaftswlssenschaft won der Kameralistlk zur Nationalokonomie" "Vortrag vor dem Dogmenhlstortschen Ausschuss des Vereins für Socialpolitik in Basel am 25. und 28. März 1982.

19 Siehe Heinz Rieter, "Quesnays Tableau Econontque als UhrenAnalogie". Erwetterte Fassung des Referats, das am 13. März 1986 in Stuttgart auf der Tagung des dogmenhistorischen Ausschusses des Vereins für Soclalpolitlk gehallen wurde. Verglefche die ABblldungen auf Seite 20 und 21 des niederländischen Textes. 
Flnanzw senschaft in vergangenen Jahrhundert bestimten; gemerngultig: festlegen, so wrd man stcherlich Ricardos (1772-1623) Princtples of Politycal Economy and Taxation (1817) und Adolph Wagners (1035-1917) Finanzwissenschaft $(1883)^{3}$ in de engere wall ziehen müsen. Der unterschted zwishen belden werken könnte nicht growser sevin. Zwar versuchen belde, die Flnanzwissenschaft systematisch in die vollowirtscheftslehre threr Zelt elnzubetten. Das wird bet Ricardo schon im Aufbau deutilch; etwa die Halfte der zwelundareissig kapitel ist der Finanzwissenschaft gewldmet. abrer nicht in der bel den Klasikern übltchen Fom, de die Finanzwissenschaft oft als ein letztes Buch (z.B. bel smth Buch W) an den Schluss stellte; bei Nicardo durchziehen de Steuern und staatsschulden des gesante Werk. Wagner dagegen trent sorgfiltig die allgemelne polltische bkononte von der finanzwissenschaft, un diese mit ihren besonderen Institutionen zur Geltung komen zu lassen.

Auffallend ist auch der Unfang. Wagners vierbändlge Flnanzwissenschaft (ln fünf Bänden) scheint sein Gesetz der wachsenden Ausdehnung der offentlichen Thätigkelten" (\$ 36) geradezu augenfällig zu bestätigen. Noch stärker frelllich springt ins Auge, wie Wagner seine Flnanzwissenschaft aufbaut. Der erste gewlchtige Band beginnt mit elner Einleltung. bespricht dam die Ordnung der Finanzwissenschaft und den Finanzbedarf und geht zugleich zum Privaterwerb des Staates über, der den grössten Tell des Buches ausmacht. Ole Bände zwey bis vier sind dann der" Stewerlehre gewidmet. Der dem Privaterwerb gewldmete Tefl bespricht zu Beginn Jewells die Grundsätze der Verstaatlichung und der Privatislerung, und geht danm auf alle öffentlichen Untepnehmen eim, die mit dem Finanzbedarf des States Im zusamenhang stehen; in diesen zusammenhang stossen wir auch auf die statsbanken und die statsschuldenbewirtschaf tung.

Zu diesem Thema vermehnen wir bef Ricardo das folgende.

From what I have sald, it must not be inferred that I consider the system of borrowing as the best calculated to defray the extraordinary expenses of the State. It is a system which tends to make us less thrifty - to blind us to our real situation ... a country Which has accumulated a large debt is placed in a most artifictal sttuation; and although the amount of taxes and the increased 
price of labour, may not, and I believe toes not, place it under any other disadvantage with respect to foreign countries, except the unavolidable one of paying those taxes, yet it becomes the interest of every contrlbutor to withdraw his shoulder from the burthen, and to shift this payment from himself to another; and the temptation to remove himself and his capltal to another country, where he w11 be exempted from such burthens, becomes at last irresistable, and overcomes the natural reluctance which every man feels to quit the place of his birth and the scene of his early associations. A country which has involved Itself in the difficulties attending this artificial system would act wisely by ransoming itself from them at the sacriftce of any portion of its property which might be necessary to redeem its debt. 20

Dles ist das klassische Plädioyer für die Lösung des Staatschuldenproblems durch Privatislerung öffentlicher Vermögen.

Der kurze Uberblick über Wagners Aufbau seines finanzwissenschaftlichen Lehrbuches lehrt uns, dass Ricardos Rat nicht allenthalben befolgt wurde, viellefcht, weill er nicht befolgt werden konnte. Wir erinnern uns, dass Ricardo die statalichen Entscheidungsträger nicht zentral in die Analyse miteinbezieht. Wle in der klassischen Tradition üblich, wird der Staat vom Markt getrennt. Die Analyse folgt ausschliesslich volkswirtschaftlichen Gesetzmässigkeiten, nicht den Eigengesetzlıchkeften staatlicher Institutionen. Tatsächlich liegt zwischen den Publikationsdaten der beiden wichtigen klassischen Werke dfe Epoche der grossen Staatsbanken auf dem europäischen Kontinent. Die öfentiliche Finanzpraxis der nach-Napoleonischen zeit stand in vielen mitteleuropässchen Staaten in schroffestem Gegensatz zu Ricardos Empfehlungen, gerade weil das Ausmass der Statsschulden einen Umfang angenamen hatte, der efn Privatisterungsprogram gar nlcht mehr zulless. Die Preussische Seehandlung (gegrundet 1772) hatte befspleiswelse 1804 gegen $4 \%$, später $3 \%$, ohne besondere Staatsgarantien von privater Selte beinahe 25 Milltonen Taler aufgenommen, während thre Aktiwa vor allem aus Preussischen Staatspapteren bestanden, die 1806 nlcht eingelöst werden konnten. Statt zu liquldieren erhlelt die Bank 
1610 statsgarantien und fungterte fortan sowohl als staltsank als ach als Industrieentwcklungsamk, mit Aktwitaten im stadilichen Strassenbau, im Elsenbahn wh schlffahrtswesien, fm Woll- und Salzhandel, Muntenwesen, der Malshtnenweberel, papterfabriken, Maschinembau und Seeschiffahrt. Da micht die diese Untermehnungen glüklich verllefen, gehörte es zur Geschaftspraxisi, unrentable Betrlebszwelge sthnell wieder abzustossen. Heben der nicht elnfachen Bewirtschaftung der kelneswegs unerheblichen Staatsschulden erfülice das Institut insofern auch wesentilche Aufgaben in der industrlellen Entwitiktung und es gelang daruberhlnaus, Jahrllich namhafte Uberschusse (im Umfange von etwa 2 Promillle der Netto Staatseinnahmen) an den Staathiaushalt fliessen zu iassen. In diesem Zusammenhang ist es wichtig zu erwathen, dass dte seehandlung selt 1810 uber keinerled Monopulrechte verfügte. 
Uber die Bedeutung der unternehmerischen Staatseinnahmen Im Vergleich zum schuldendienst informlert instruktiv die folgende Tabelle für das Jahr 1900 .

$\begin{array}{llll}\text { Bruttobedarf } & \text { Uberschüsse } & \text { Wenfger } & \text { Prozentverhält- } \\ \text { der } & \text { der sog. pri- } & \text { oder Mehr } & \text { nis zwischen } \\ \text { Verzinsung } & \text { vatwirt- } & \text { Nettobedarf } & \text { Uberschüssen } \\ & \text { schaftilichen } & \text { der } & \text { U.d. Bruttobe- } \\ & \text { Elnnahmen } & \text { Verzinsung } & \text { darf der Ver- } \\ \text { Mi11.M. } & \text { M111.M. } & \text { M111.M. } & \text { zinsung (\%) }\end{array}$

\begin{tabular}{|c|c|c|c|c|}
\hline Deutsches & 77,700 & 72,833 & 4,867 & 9,37 \\
\hline \multicolumn{5}{|l|}{ Reich } \\
\hline Preusisen & 272,751 & 623,045 & $=350,294$ & 228,40 \\
\hline Bayern & 49,394 & 70.670 & 21,276 & 143,10 \\
\hline Wïrtbg. & 20,204 & 26,481 & 6,277 & 131,10 \\
\hline Sachsen & 35,260 & 50,088 & $14,8.28$ & 141,80 \\
\hline Ost.-Ung. & 214,281 & - & $+214,281$ & - \\
\hline Ost. & 129,672 & 106,804 & 22,768 & 82,37 \\
\hline Ungarn & 160,228 & 155,247 & 4,981 & 96,89 \\
\hline Frkr. & 1002,124 & 30,834 & $+971,290$ & 3,08 \\
\hline Itallen & 471,277 & 82,460 & $+\quad 388,817$ & 17,50 \\
\hline England & 464,332 & 13,080 & $+451,252$ & 2,82 \\
\hline Russland & 590,661 & 482,614 & $+107,947$ & 81,84 \\
\hline
\end{tabular}

Quelle: Max von Heckel, "Staatsschulden". In: Handwörterbuch der Staatswissenschaften, Jena: Gustav Fischer, 1901, Band 6, S. 968.

Diese Zahlen belegen eindrücklich die Unterschlede fm Finanzgebaren einzelner Länder, vor allem fm Hinblick auf die privatunternehmerische Staatstätigkeit; dem entspricht folgerichtig die unterschiediche Schwerpunktsetzung etwa bel Ricardo und Wagner.

Dle unterschiedlichen Wirtschaftsstlle, für dfe die Flnanzwissenschaftler Wagner etnerseits und Ricardo andererselts stehen, lassen slch noch an einem anderen Aspekt der Staats schuldenproblematik demonstrleren. In seiner berühten Aquivalenztheorle postulfert Ricarda, dass eine Zunahme der Staatsschuld auch zu einer Zunallme der erwarteten Steuerschuld führt und infolgedessen zul eliner ebenso hohen Zunahme der privaten Ersparnisse, um die erwartete höhere steuerschuld aufzu- 
fangen, 21 Wagner $15 t$ demgegenuber der Auffassung, dass eine Zunahme der zu erwartenden steuerschuld als folge elrer Erhöhung der Statsschuld nicht ohne weiteres postullert werden kann. Die Erwartungem hinstchtllch der Stewerschuld hängen vor allem davon $a b$, wie die durch die Schuldenaufrahme gewomenen Hittel eingesetzt wurden. Das schilesst dann slcher die Mögllchkelt ein, dass über den Kapltalmarkt fuanzlerte offentiliche Investitionen, die selbst weder Ertrage abwerfen, zu einer Senkung der erwarteten Steuerschuld führen können.

Unsere elgenen Berechnungen mit amerikanischem Daten haben gezelgt, dass die Wahrhelt, we so oft, etwa in der Mitte liegt. Eine Zunahme des Staatsvermögens um elnen Dollar, dfe aus laufenden Staatsausgaben finanzitert wird, fuhrt zw efiner Senkung der privaten Ersparnisse um slebenundfunfzlg Cents. 22 Daraus könnem wir den Schluss ablelten, dass amerlkanfsche Bürger erwarten, dass ungefähr die Hälfte der öffentlichen Inwestitionen produktiv vorgenomen werden, die andere Hälfte eher konsumptiven Charakter hat. Nur diese letzteren müssen durch Stevern aufgefangen werden.

\section{Schllussfolgerung}

Für mein eigenes Fach zitehe fch daraus den Schluss, dass es an uns llegt, gute Finanzwissenschaftler auszubilden, die in threr beruflichen Praxis wlllens und in der Lage sind, staatlifche Gelder produktiv einzusetzen und $\mathrm{mit}$ Gewinn arbeften zu lassen. Eln so der kameraIistischen Tradition verpflichtetes offentliches Ausgabeverhalten müste zu der rationalen Erwartung führen, dass eine Stelgung der öffentlichen Schuld zur Finanzierung öfentlicher Projekte eine Steuerlasterlefichterung erlaubt, wenn und solange das öfentliche Investitionsverhalten darauf gerichtet ist, die Finanzkraft der äffentllichen Hand zu stärken.

21 Ricardo postullert gleichzeitlg auch die Non-Aquivalenztheorie, und lässt deren Gültigkeft von der form abhängen, die die Steuer annimt. Dleser Zusammenhang ist für das folgende nicht von Bedeutung und wird deshalb ausser Acht gelassen.

22 Vergleiche im einzelnen Jürgen Backhaus, Randall Holcombe, Asghar Zardkoohi, "Public Investments and ilts Effects on the Burden of the Public Debt"ll, Southern Economlc Journal, 54.1, 1987, S. 154-158. 

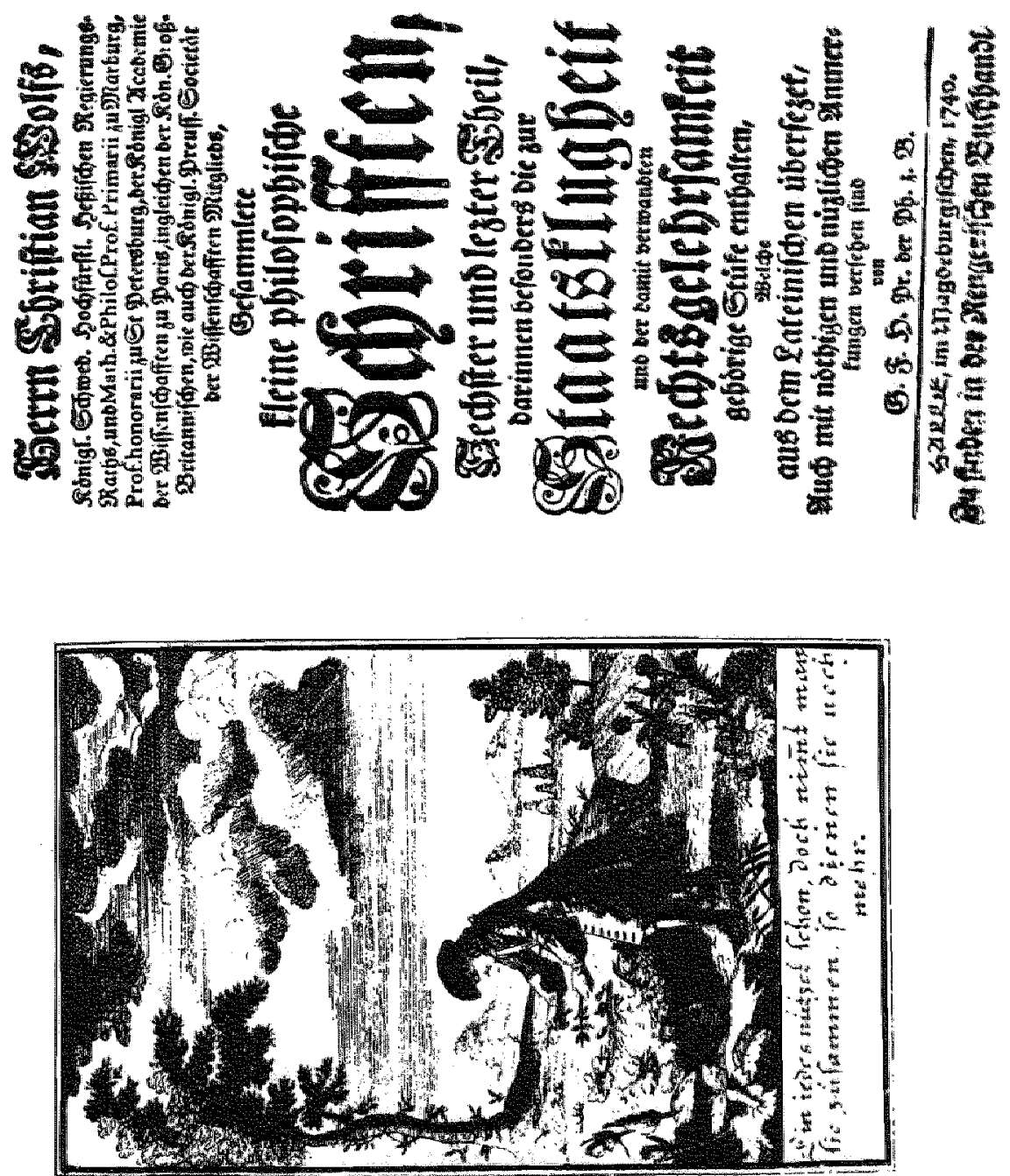